\title{
Nano materials applications for conservation of cultural heritage
}

\author{
A. M. Waked \\ Department of Architecture, Modern Academy for Engineering \& \\ Technology, Cairo, Egypt
}

\begin{abstract}
Nanotechnology is considered to be the most important theoretical and applicative framework of human knowledge in the near future, breakthroughs are restricted to few applications. One is being the conservation and restoration of cultural heritage. For the architecture profession nanotechnology will be able to change the way we build, and will greatly impact construction materials and their properties as well. However, materials will behave in many different ways as we are able to more precisely control their properties at nano-composites, which combine new nano-materials with more traditional ones and can be many times stronger than standard materials, and will dramatically improve the performance, durability, and strength to weight ratio of these materials.

As materials gain such transient features architectural design and construction will evolve by transforming the essential properties of matter.

This study of material is considered an emerging field, so this paper will retry to represent the most recent applications of nano materials of the conservation of cultural.
\end{abstract}

Keywords: nanotechnology, nano-materials, conservation.

\section{Introduction}

The use of nanotechnology in architecture is wide and varies from the early stages of sketching up to the final touches of finishing, especially in choosing the right material which will not only reflect the design but also has a great influence in the methodology of thinking of architecture according to the new vast options. Nano architecture is the integration of nanotechnology in architecture, by using nano-products, nano-materials or even nano-shapes. One of nanotechnology's 
applications is the conservation of cultural heritage Wall paintings conservation and paper de-acidification have recently provided clear evidences of the huge potentiality of this emerging science for cultural heritage conservation.

\section{Nanotechnology and architecture}

\subsection{The definition of nano}

The term "nano" derives from The Greek word for dwarf [1]. It is used as a prefix for any unit; hence a nanometer $(\mathrm{nm})$ is a billionth of a meter or 10-9 meter [2].

\subsection{The definition of nanotechnology}

Nanotechnology can be difficult to determine and define. In fact, a committee was formed (the National Nanotechnology Initiative) and the following defining features of nanotechnology were hammered out [3]:

1-Nanotechnology involves research and technology development at the $1 \mathrm{~nm}$-to- $100 \mathrm{~nm}$ range.

2. Nanotechnology creates and uses structures that have novel properties because of their small size.

3. Nanotechnology builds on the ability to control or manipulate at the atomic scale. Generally Nanotechnology involves developing materials or devices within that size. Nanotechnology is very diverse, ranging from extensions of conventional device physics to completely new approaches based upon molecular self-assembly, from developing new materials with dimensions on the Nanoscale to investigating whether we can directly control matter on the atomic scale [4].

The "nanoscale" (see Figure. 1) is typically measured in Nanometers, or billionths of a meter, and materials built at this scale often exhibit distinctive physical and chemical properties due to quantum mechanical effects. Techniques for working at the nanoscale have become essential to electronic engineering, and nanoengineered materials have begun to appear in consumer products. For example, billions of microscopic "nanowhiskers," each about 10 nanometers in length, have been molecularly hooked onto natural and synthetic fibers to impart stain resistance to clothing and other fabrics; zinc oxide Nanocrystals have been used to create invisible sunscreens that block ultraviolet light; and silver [5].

There are two main approaches are used in Nanotechnology [2, 3]:

1-The "bottom-up" approach where materials and devices are built from molecular components which assemble themselves chemically by principles of molecular recognition.

2-The "top-down" approach; nano-objects are constructed from larger entities without atomic-level control. 


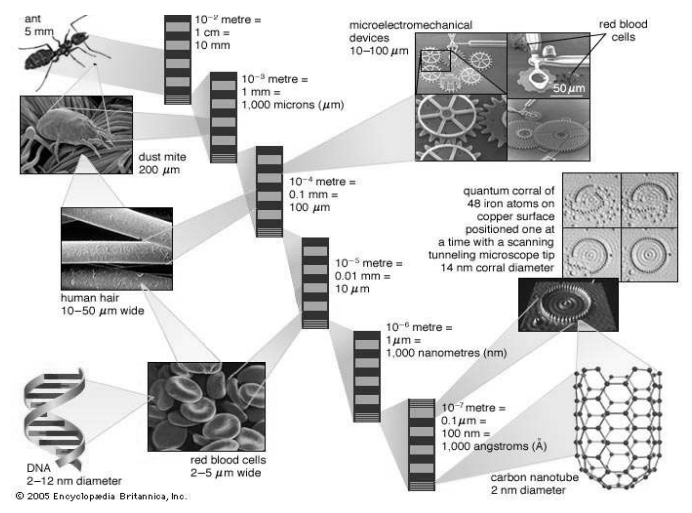

Figure 1: Examples from biological and mechanical realms illustrate various "orders of magnitude" (powers of 10 ), from $10^{-2}$ metre down to $10^{-7}$ metre.

\subsection{Nano architecture}

Nanotechnology will impact environments. It will give architecture superior interactive functions - allowing occupants to better communicate with their surroundings. As new materials and construction methods emerge, "Nano Architecture" will definitely unleash the designer's imagination [6].

\subsubsection{Nanoarchitecture definition}

Nanoarchitecture is the conversion of architecture in the new nano revolution in the $21 \mathrm{st}$ century. The use of nanotechnology in architecture varies from materials, equipments, forms and design theories [7].

\section{Architecture and nanomaterials}

In architecture two fundamentally different design approaches prevail when dealing with materials and surfaces:

A-Honesty of materials - "what you see is what you get," such as natural stone or solid woods.

B-Fakes - artificial surfaces that imitate natural materials. In future, a third option will be available.

C-Functional nanosurfaces, emancipated from underlying materials.

The properties of such ultra-thin surfaces can differ entirely from the material they enclose and can be transparent and completely invisible. Also possible are nanocomposites with new properties: nanoparticles or other nanomaterials are integrated into conventional materials so that the characteristics of the original material are not only improved but can be accorded new functional properties or even be made multifunctional. Surface materials that are customized to have specific functional properties are set to become the norm, heralding a switch 
from catalogue materials to made-to-measure materials with definable combination of properties - a perfectly modular system.

\subsection{Nanomaterials}

The paper studies materials with morphological features on the nanoscale, and especially those which have special properties stemming from their nanoscale dimensions. Nanoscale is usually defined as smaller than a one tenth of a micrometer in at least one dimension, though this term is sometimes also used for materials smaller than one micrometer [8]. A nanomaterial is an object that has at least one dimension in nanometer scale. Common types of nanomaterials include nanotubes, dendrimers, quantum dots and fullerenes [10].

Table 1: $\quad$ Nanomaterials categorized based on dimension.

\begin{tabular}{|l|l|l|}
\hline $\begin{array}{l}\text { Nanomaterial } \\
\text { Dimension }\end{array}$ & Nanomaterial Type & Example \\
\hline $\begin{array}{l}\text { All three dimensions }< \\
100 \mathrm{~nm}\end{array}$ & $\begin{array}{l}\text { Nanoparticles, Quantum dots, } \\
\text { nanoshells, nanorings, } \\
\text { microcapsules }\end{array}$ & Nanotubes, fibres, nanowires \\
\hline $\begin{array}{l}\text { Two dimensions }<100 \\
\text { nm }\end{array}$ & Thin films, layers and coatings \\
\hline $\begin{array}{l}\text { One dimension }<100 \\
\text { nm }\end{array}$ & \\
\hline
\end{tabular}

\subsubsection{Classification of nanomaterials}

Materials referred to as "nanomaterials" generally fall into two categories: fullerenes and inorganic nanoparticles.

\section{a) Fullerenes}

The fullerenes are a class of allotropes of carbon which conceptually are graphene sheets rolled into tubes or spheres. These include the carbon nanotubes (or silicon nanotubes) [11].

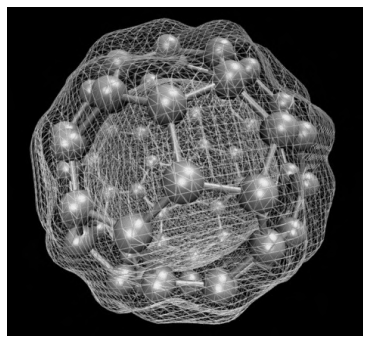

Figure 2: $\quad$ C60 with surface of ground state electron density as calculated with DFT. 


\section{b) Inorganic nanoparticles}

Nanoparticles or nanocrystals made of metals, semiconductors, or oxides are of particular interest for their mechanical, electrical, magnetic, optical, chemical and other properties. Nanoparticles are of great scientific interest as they are effectively a bridge between bulk materials and atomic or molecular structures. A bulk material should have constant physical properties regardless of its size, but at the Nano-scale this is often not the case [12].

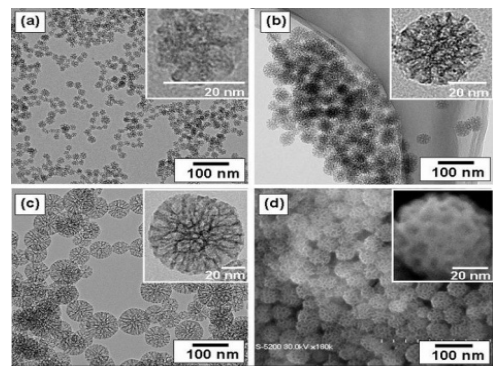

Figure 3: TEM (a), (b), and (c) images of prepared mesoporous silica nanoparticles with mean outer diameter: (a) $20 \mathrm{~nm}$, (b) $45 \mathrm{~nm}$, and (c) $80 \mathrm{~nm}$. SEM (d) image corresponding to (b). The insets are a high magnification of mesoporous silica particle.

\subsection{Nano material products}

There are several material products such as: coating, insulation, air purifying, solar protection [13].

Coating in order to produce a layer which is bound to the base material to produce a surface of the desired protective or functional properties. Research is being carried out through experiment and modeling of coatings and one of the goals is the endowment of self healing capabilities through a process of "selfassembly" [14].

Nanotechnology is being applied to paint and insulating properties, produced by the addition of nano-sized cells, pores and particles, giving very limited paths for thermal conduction ( $\mathrm{R}$ values are double those for insulating foam), are currently available. This type of paint is used, at present, for corrosion protection under insulation since it is hydrophobic and repels water from the metal pipe and can also protect metal from salt water attack [14]. In addition to the self-cleaning coatings mentioned above for glazing, the remarkable properties of $\mathrm{TiO}_{2}$ nanoparticles are being put to use as a coating material on roadways in tests around the world. The $\mathrm{TiO}_{2}$ coating captures and breaks down organic and inorganic air pollutants by a photo-catalytic process (a coating of $7000 \mathrm{~m}^{2}$ of road in Milan gave a $60 \%$ reduction in nitrous oxides).

\subsubsection{Self-cleaning: Lotus-Effect [13]}

- Microscopically rough, not smooth.

- Hydrophobic - water trickles off. 
This is one of the best-known means of designing surfaces with nanomaterials. The name "Lotus-Effect" is evocative, conjuring up associations of beads of water droplets, and therefore the effect is often confused with "Easyto-clean" surfaces or with photo-catalysis, which is also self-cleaning.

Here is an example for the use of the self-cleaning Lotus Effect applied on a building surface for a better optimal use and low maintenance façades:

\section{Ara Pacis Museum, Rome, Italy, 2006}

After ten years of construction and political debate, the Ara Pacis Museum is now home to an archaeological highlight in Rome. The Ara Pacis Augustae, a sacrificial altar that was inaugurated by the Emperor Augustus himself, was given a new container that remedies the environmental as well aesthetic deficits of the previous pavilion from 1938 in which the monument was formerly kept. A tripartite building complex has been created on the banks of the River Tiber, whose urban form now fits in with the historic centre of Rome, and which connects antiquity with the modern.

Here a self-cleaning coating has been invisibly integrated into the white surfaces to ensure the durability of their color. In the heavily polluted city, it would not otherwise have stood much chance of remaining white for long.
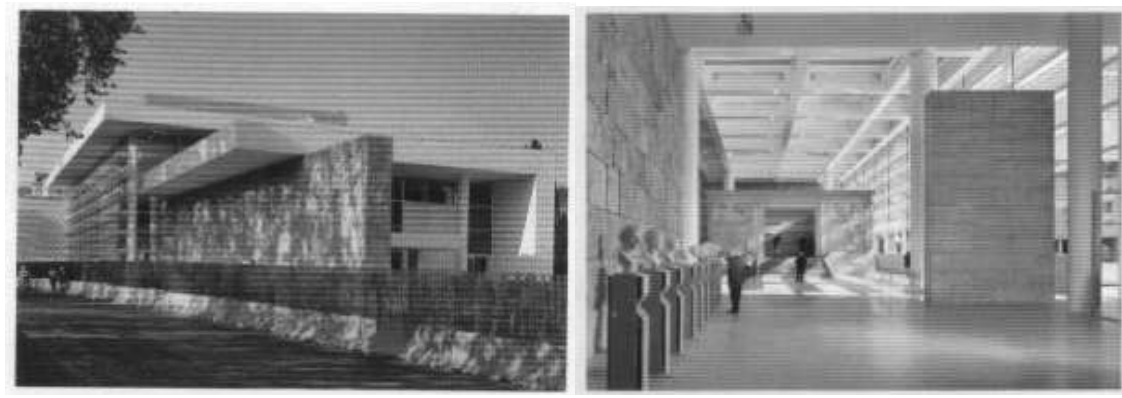

Figure 4: $\quad$ Ara Pacis Museum with self cleaning coating.

\subsubsection{Self-cleaning: photo-catalysis}

- Hydrophilic surfaces.

- Deposited dirt is broken down and lies loose on the surface.

- A water film washes dirt away.

- UV light and water are required.

- Reduces maintenance requirement.

Photocatalytic self-cleaning is probably the most widely used nano-function in building construction, with Japan leading the field. There are numerous buildings of all sizes around the world that make use of this function. Its primary effect is that it greatly reduces the extent of dirt adhesion on surfaces. It is important to note that the term "self-cleaning" in this context is misleading and does not mean, as commonly assumed that a surface does not need be cleaned at all. Fewer detergents are required, resulting in less environmental pollution and less wear and tear of materials. Likewise, reduced cleaning cycles lead to savings 


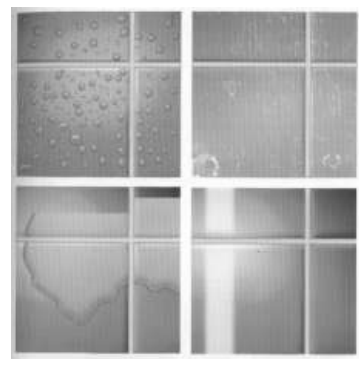

Figure 5: Before and after: on conventional tiles water forms droplets that dry leaving behind dirt deposits. On the hydrophilic surfaces of photocatalytic tiles, water forms a film that runs off taking any loose dirt deposits with it.

in personnel costs and the fact that the dirt adheres less means that it is also easier to remove.

Generally speaking, photo-catalytic self-cleaning is a low-maintenance and trouble-free solution. A further advantage is that light transmission for glazing and translucent membranes are improved as daylight is obscured less by surface dirt and grime. Energy costs for lighting can be reduced accordingly.

For the function to work, UV light, oxygen and air humidity are required (see Figure 6). The level of UV light present in normal daylight is sufficient to activate the photo-catalytic reaction. Organic dirt on the surface of a material is decomposed with the help of a catalyst - usually titanium dioxide $\left(\mathrm{TiO}_{2}\right.$, and the particularly reactive derivative Anatase). The nanoscale dimension of $\mathrm{TiO}_{2}$ makes it a highly reactive catalyst, speeding up the decomposition process rapidly without being used up so that the effect is lasting.
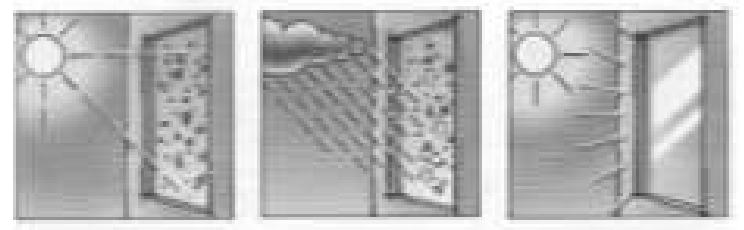

Figure 6: The diagram shows the basic process: Organic dirt and grime is broken down and "decomposed". Until now UV light, such as present in sunlight, is necessary to initiate photocatalysis. When water impacts on the surface, it spreads to form a film washing away the loose dirt. The result: clean surfaces.

In addition to the catalyst, the UV component of light, with a wavelength of less than $390 \mathrm{~nm}$ is considered essential for the reaction to occur, and its intensity plays an important role. As such, photo-catalytic self-cleaning surfaces are generally speaking more effective outdoors than indoors. The method is predestined, for example, for use on building façades. 
The hydrophilic properties of Ti02 were discovered much later. Due to its increased surface energy such surfaces are hydrophilic (water-attracting), which means that water runs off from any inclined surface in a film rather than in droplets (see Figure 7 ). In comparison to Lotus-Effect surfaces, this coating is transparent and can be applied to glass invisibly. Photo-catalytic surface coatings are often applied to façade panels made of glass or ceramics or to membranes.

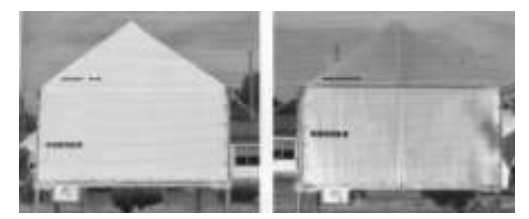

Figure 7: $\quad \mathrm{TiO}_{2}$ and PVC coated white membranes in weathering tests. The difference is readily apparent: after 5 months the former is still white, the latter grey and unsightly.

In production it is only economical for mass-produced glass as the coating is usually applied in the factory using chemical vapor deposition (CVD), a vacuum coating technique in which an ultra-thin coating is applied in vapor form. Such coatings cannot be retrofitted. However, this does not limit its application exclusively to large buildings; it can be equally appropriate for example for conservatories and winter gardens.

In road buildings the transparent coating can also be used, for example, for noise barriers. Tiles with baked-on durable coatings are available for use both indoors and outdoors. Likewise, concrete, can also be equipped with a selfcleaning surface.

An interesting application is the exploitation of the cooling effect of evaporating water. The canvas and steel roofing as well as the windows of a trade fair pavilion in Japan (Expo 2005) were equipped with a photo-catalytic $\mathrm{TiO} 2$ coating and subjected to a constant stream of water. Duo to the hydrophilic property of the surface, the water immediately formed a thin film which evaporates quickly absorbing in the process ambient warmth and thereby reducing the indoor temperature. Initial estimates suggest a potential energy reduction of between $10-20 \%$ in comparison to conventional air conditioning.

\section{Narita International Airport of Tokyo Terminal 1 Chiba, Japan, 2006}

In 2006, The Narita International Airport underwent comprehensive renovation. In the process large areas were covered with textile. Membranes offer protection against the weather and therefore improving comfort for the passengers. As the membranes are equipped with a photocatalytic self-cleaning coating, the cost of cleaning and maintenance is kept to a minimum. In central areas of Tokyo, the use of self-cleaning awnings has been common practice for several years and they have proven to remain much cleaner than their conventional counterparts. Although conventional surface coating, glass, PTFE or ETFE materials are also self-cleaning, they are not able to stop dirt deposits from accumulating. 

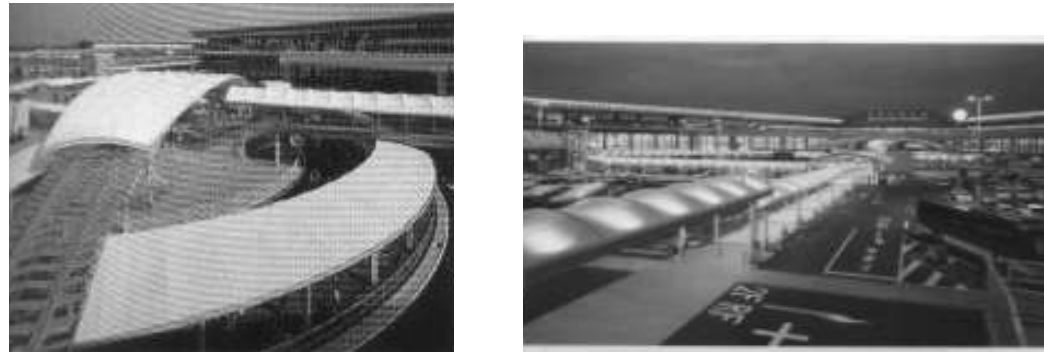

Figure 8: Narita International Airport with photocatalytic self clean.

\subsubsection{Easy-to-clean (ETC) [13]}

-Smooth surfaces with reduced surface attraction.

-Surface repellence without using the Lotus-Effect.

Easy-to-clean (ETC) surfaces are water repellent and accordingly are often confused with other self-cleaning functions such as the Lotus-Effect. However, unlike the latter, easy-to-clean surfaces are smooth rather than rough. These surfaces have a lower force of surface attraction due to a decrease in their surface energy, resulting in reduced surface adhesion. This causes water to be repelled, forming droplets and running off. Easy-to-clean surfaces are therefore hydrophobic, and often also oleophobic. This function is used for coating ceramic sanitary installations and shower cubicle glazing. Wood, metal, masonry, concrete, leather as well as textiles are likewise candidates for hydrophobic coatings. Easy-to-clean surfaces are less susceptible to dirt accumulation (dirt repellent). The benefit: stress-free and easy cleaning saves time and costs. Here is an example for the use of the Easy To Clean (ETC) applied on a building surface for a better optimal use and low maintenance façades.

\section{Kaldewei Kompetenz-Center (KKC), Ahlen, Germany, 2005}

Kaldewei is unique among bath manufacturers in that it has its own in-house enamel development and production facilities. By wrapping its building in a veillike facade of colored steel-enamel panel elements, behind which the existing

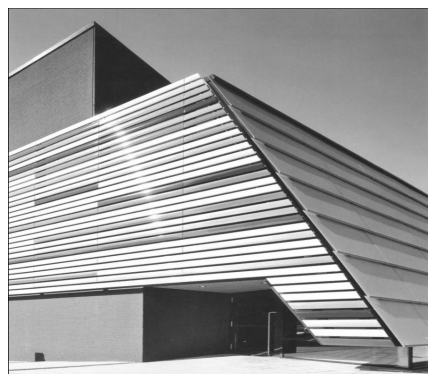

Figure 9: The enameled facade panels of Kaldewei Kompetenz-Center (KKC). 
melting facilities can be seen, the company expresses its brand through architecture. The enameled facade panels are colored in the company's typical color palette and are partially equipped with an easy-to-clean coating.

This coating is otherwise used in the manufacture of bathtubs to further improve the ease with which one can clean the already low-maintenance material.

\section{Nano and conservation of cultural heritage}

Although nanotechnology is considered the most important theoretical and applicative framework of human knowledge for the near future, breakthroughs are restricted to few applications, one being the conservation and restoration of cultural heritage. The most recent applications of nanotechnology to the conservation and restoration of the world cultural heritage are discussed in this review and some specific applications of humble calcium and magnesium hydroxide and carbonate nanoparticles, showing their efficacy for the restoration and protection of wall paints and for the de-acidification of paper and wood, are highlighted [15].

Aside from the enormously rich cultural resources in the city of Florence, it is one of the most suitable places for conservation studies. For example, after the1966 Florence flood, the Center for Colloid and Surface Science (CSGI) research group at the University of Florence, founded by Prof. Enzo Ferroni and currently directed by Piero Baglioni, was the first academic institution that applied a rigorous scientific approach to the investigation of cultural heritage degradation CSGI has developed the most advanced nanotechnology-based methods for the restoration of wall paintings. These include methods for cleaning and removal of resins from wall and oil paintings, for frescoes consolidation, and for paper de-acidification. Currently these methods are used in many parts of the world.

Applications of nanotechnology-based processes to wall paintings consolidation and paper de-acidification have recently provided clear evidences of the huge potential of nanotechnology for cultural heritage conservation. Nano dispersions of solids, micelle solutions, gels and microemulsions offer new reliable ways to restore and preserve works of art by merging together the main features and properties of soft-matter and hard-matter systems, allowing the synthesis of systems specifically tailored for the works of art to fight the deterioration processes which threaten many priceless masterpieces. Several conservation workshops have been carried out with CSGI scientific consultancy, and using innovative methodologies developed in the CSGI Laboratories (Masaccio's wall paintings in Cappella Brancacci, and Beato Angelico's wall paintings in San Marco Abbey, in Florence, Piero della Francesca's wall paintings in Arezzo, etc.) [16]. 

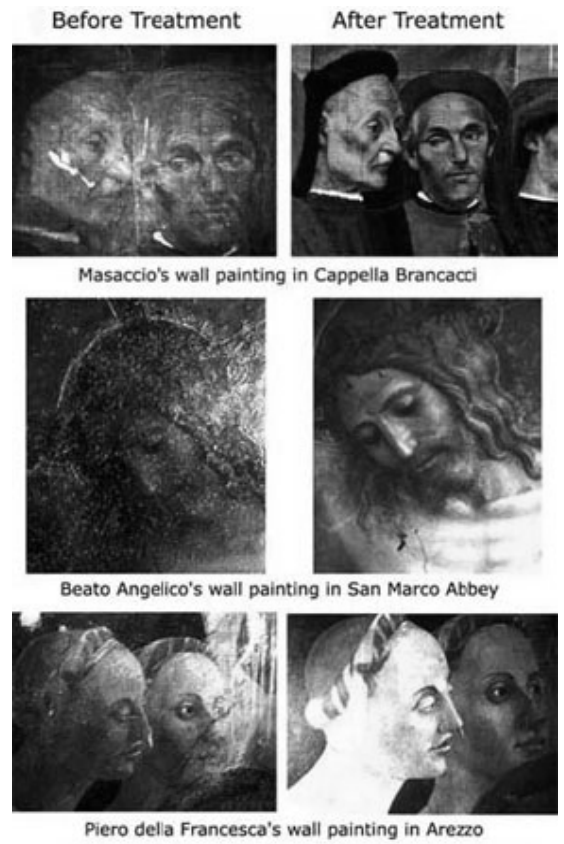

Figure 10: The difference between pre- and post-restoration.

\section{Conclusion and recommendation}

- Nano-technologies enable the design and production of agents highly compatible with historic materials, with very high chemical effectiveness due to their increased surface-to-volume ratio and a higher concentration of active substances, without side-effects.

- Nano-materials play an important role in conservation of art and architectural objects because they capable of responding to the need to preserve the maximum amount of the original substance, due to their ability to penetrate into various and variable porous systems.

- Nano-materials are used advantageously for consolidating disintegrated historic materials, materials with cracks and fissures, materials with complex problems, namely wet and chemically contaminated materials, or in situations requiring complex solutions.

- The study for the better by using nano material to achieve sustainability, and conservation in buildings' exteriors and Interiors.

\section{References}

[1] Kathleen A. Cook, Discover Nano: Nano 101, Northwestern University, 2005 Retrieved Feb. 2011 from(http://www.discoverNano.northwestern.ed) 
[2] Michael F. Ashby, Paulo J. Ferreira and Daniel L. Schodek "Nanomaterials, NanoTechnologies and Design", Linacre Haus, Jordan Hill, Oxford , 2009

[3] Richard \& Boysen, Earl. Wiley, Nanotechnology for the Dummies, The Hitchhiker's Guide to Nanotechnology. Bookers, Publishing Inc. 2005.

[4] Nanotechnology: A Brief Introduction", Interdisciplinary Nanoscience Center (INANO) University of Aarhus, Denmark., Retrieved April 2011 from (http://naocap.eu/flex/site/download.aspx

[5] "Nanotechnology", Encyclopaedia Britannica, 2005. Retrieved April 2011.from http://www.britannica.com/EBchecked/topic/Nanotechnology

[6] Cathryn Bang, "Intersecting of Nanotechnology Materials for Green Buildings", Healthcare Architecture Planning Interiors, New York, Retrieved, May 2011, from www.cbparch.com

[7] Maged Fouad Al Samny," NanoArchitecture, Nanotechnology and Architecture", unpublished Master Research, Architecture Dept, Alex. University, 2008

[8] "NanoMaterial" Retrieved Aug 2010 from http://en.wikipedia.org /Nanomaterials

[9] Lusia Fillpponi \& Duncan Sutherland "Nanomaterial Science Nanotechnology”, 2007, retrieved Aug 2010 from http://en.wikipedia.org /wiki/Nanotechnology

[10] Nanomaterials: NICNAS Information Sheet, Australian Government Department of Health and Ageing Nicnas, Sept.2006, retrieved August 2010 from http://nicnas.gov.au/public/information_sheets/general_ information_sheets/nis_Nanomaterials_pdf.pdf

[11] "Fullerenes", retrieved Aug 2010 from (http://en.wikipedia.org /wiki/Fullerene)

[12] "InorganicNanoparticles", retrieved April 2011 from: (http://en.wikipedia.org/wiki/Nanoparticl)

[13] Leydecker Sylvia, "Nano Materials in Architecture, Interior Architecture and Design". BirkhauserVerlag AG , 2008

[14] Surinder Mann, Nanoforum report "Nanotechnology and Construction, European Nanotechnology Gateway", 2006,Nanoforum.org retrieved Apr 2011 from (http://Nanoforum.org)

[15] Piero Baglioni and Rodorico Giorgi., "Soft and Hard Nanomaterials for Restoration and Conservation of Cultural Heritage", 2006, retrieved April 2011 from (www.rsc.org/softmatter)

[16] Baglioni, P., R. Giorgi and C. C. Chen, "Nanoparticle technology saves cultural relics, and potential for a multimedia digital library", DELOS/NSF Workshop on Multimedia Contents in Digital Libraries, Crete, Greece, 2003 